\title{
The prognostic importance of double-expressor subgroup and AID, UNG and mismatch repair protein expressions in diffuse large B-cell lymphomas
}

\author{
Muhammed Hasan TOPER ${ }^{1}$ (D), Suheyla BOZKURT² (D), Tayfun ELIBOL ${ }^{3}$ (D), Tulin TUGLULAR ${ }^{3}$ (D) \\ 1 Department of Molecular Pathology, Dokuz Eylul University Graduate School of Health Sciences, Izmir, Turkey. \\ 2 Department of Pathology, School of Medicine, Marmara University, Istanbul, Turkey. \\ 3 Sub-department of Hematology, Department of Internal Medicine, School of Medicine, Marmara University, Istanbul, Turkey. \\ Corresponding Author: Muhammed Hasan TOPER \\ E-mail: hasantoper@gmail.com
}

Submitted: 21.02 .2020

Accepted: $\quad 07.04 .2020$

\section{ABSTRACT}

Objective: The cases of diffuse large B-cell lymphoma, (DLBCL not otherwise specified (NOS)) which immunohistochemically exhibit MYC and BCL2 expressions are defined as double-expressor lymphomas (DELs). This study aimed to assess the prognostic impact of DEL and the expressions of other proteins that may have role in tumorogenesis.

Materials and Methods: In this study, 90 tumor samples from patients diagnosed with DLBCL NOS were evaluated retrospectively. Immunoexpressions of MYC, BCL2, activation-induced cytidine deaminase (AID), uracil-DNA glycosylase (UNG) and DNA mismatch repair proteins including MLH1, MSH2, MSH6 and PMS2 were analyzed.

Result: Eleven cases (12.2\%) which exhibited $\geq 40 \%$ MYC and $\geq 50 \%$ BCL2 immunexpressions were classified as DEL DLBCL. Patients with MYC positivity displayed lower overall survival rate than MYC negative cases. A trend of lower overall survival was observed in the double-expressor lymphoma group, however, this was not proven to be statistically significant. Significant relationship between AID, UNG and p53 immunexpressions with double-expressor lymphoma or overall survival was not detected. The correlation between immunexpressions of p53 and MYC was observed. The loss of expression of mismatch repair proteins was not observed in any cases.

Conclusion: In this study, a relationship between low overall survival and MYC expression is detected. However, our result does not demonstrate that double-expressor lymphoma can be associated with poor outcomes.

Keywords: Diffuse large B-cell lymphoma, Double-expressor lymphoma, MYC, BCL2, AID, Mismatch repair proteins

\section{INTRODUCTION}

Diffuse large B-cell lymphoma (DLBCL) is the most common aggressive B-cell lymphoma and a heterogenous group of diseases in terms of morphologic appearances, immunhistochemical phenotypes and molecular aberrations.

Several studies have been made with a great effort to subtype DLBCL at all levels to predict clinical outcomes and determine suitable treatment regimes. The main treatment for DLBCL not othervise specified (NOS) is the use of rituximab, cyclophosphamide, doxorubicin, vincristine and prednisone (R-CHOP). The disease recurs in nearly half of patients. In order to predict the clinical course, the National Comprehensive Cancer Network-International Prognosis Index (NCCN-IPI) score system has been established with clinical and laboratory data [1-3].

It has been reported that double-expression of MYC and BCL2 in DLBCL is a prognostic factor by earlier studies of Johnson et al. and Green et al. Numerous studies have contributed to our knowledge about these cases. DLBCL cases which show overexpression with both MYC and BCL2 antibodies with the cut-off values of $40 \%$ and $50 \%$ respectively, have been mentioned as double-expressor lymphoma (DEL), according the last World Health Organization (WHO) lymphoma classification update [3]. It is advised to identify these cases because of the fact that DEL subset in DLBCL may have the role in prognostic significance [4-8].

Translocation mutations have an important role in the lymphomagenesis process in the majority of lymphomas. It is thought that DNA fractures that occur during physiological somatic hypermutation and isotype switching in normal B-cell activation related to the formation of these mutations may play a role. Activation-induced cytidine deaminase (AID), uracilDNA glycosylase (UNG), mismatch repair proteins (MLH1,

How to cite this article: Toper MH, Bozkurt S, Elibol T, Tuglular T. The prognostic importance of double-expressor subgroup and expressions of AID, UNG, mismatch repair proteins in diffuse large B-cell lymphomas. Marmara Med J 2020:33: 83-89 doi: 10.5472/marumj.741651 
MSH2, MSH6, PMS2) and p53 proteins have roles during somatic mutation and isotype switching [9-14].

The aim of this study is to assess the prognostic impact of doubleexpression with MYC, BCL2 antibodies and the expression of other proteins that may have role in tumorogenesis.

\section{MATERIALS and METHODS}

\section{Case Selection and Clinical Data}

Tumor samples from patients who were diagnosed with DLBCL NOS between January 2007 and June 2015 and treated with $\mathrm{R}-\mathrm{CHOP}$ regimen, were searched retrospectively. The samples were obtained from Pathology archive of Marmara University Hospital. Ninety cases among 187 cases were identified who met the inclusion criteria. The inclusion criteria were: to have formalin-fixed paraffin-embedded (FFPE) tumor block and have enough tumor volume in it (more than 100 cells), samples without artefact and have the survival data. Tumors that developed as secondary to low-grade lymphoma and were primarily located at the mediastinum, central nervous system, skin, intravascular and the body cavity were exluded.

The database of 90 eligible cases was collected from clinical records of the hematology department. Age, sex and tumor location of the all cases were recorded while Ann-Arbor stage, revised (R)-IPI/NCCN-IPI scores were obtained from 58 and 52 patients, respectively.

Hematoxylin and Eosin stained pathology slides were rereviewed by one hematopathologist and one general pathologist on the basis of morphology and immunohistochemistry for the purpose of confirmation of diagnosis according to the last 2016 WHO classification [15].

This study was approved by Marmara University Clinical Research Ethics Board (Protocol number: 09.2016.578). All methods were performed in accordance with the relevant guidelines regulations.

\section{Immunohistochemistry}

Sections with a thickness of $4 \mu \mathrm{m}$ per tumor were obtained from formalin-fixed paraffin-embedded (FFPE) tumor blocks for immunohistochemical analysis. Immunohistochemical staining with MYC, BCL2, BCL6, CD10, MUM1, p53 and Ki67 antibodies was performed using standard methods on the Ventana platform (Roche, Basel, Switzerland). The proper external control tissues were used for each antibody as recommended. The percentage of cells that demonstrated staining for each antibody was recorded. The intensity of staining also was recorded and moderate or strong intesity of immunoexpression were accepted as positive staining, while weak immunoexpression was not. According the last WHO recommendation, thresholds for immunoexpression of tumor cells with MYC and BCL2 antibodies are accepted as $40 \%$ nuclear staining and 50\% cytoplasmic staining, respectively [15]. The cases that were stained with MYC and BCL2 antibodies greater than these percentages were defined as DEL. Fluorescence in situ hybridization (FISH) analyses for MYC and BCL2 were not performed.
Cell of origin (COO) was determined according to Hans algoritm with CD10, BCL6 and MUM1 antibodies and cases were grouped as germinal center B-cell like (GCB) DLBCL and non-germinal center B-cell like (non-GCB) DLBCL [16]. Ki67 proliferation index recorded in the area of tumor showed maximum percentage of staining. Cases grouped according the percentage of staining as below $50 \%$, between $50-90$ and more than $90 \%$. Nuclear staining of tumor cells for p53 antibody was evaluated and then cases were grouped as below or above 50\% staining according to p53 immunoexpressions. Immunhistochemical staining with AID, UNG and DNA mismatch repair proteins including MLH1, MSH2, MSH6, and PMS2 antibodies were also studied. Cut-off percentages for staining were accepted as $15 \%$ and $20 \%$ for AID and UNG respectively. The loss of staining with MLH1, MSH2, MSH6, and PMS2 antibodies were recorded for each tumor.

\section{Statistical Analysis}

Fisher's exact test and Pearson $\chi^{2}$ tests were used for group comparison of categorical variables. Overall survivals were calculated from the date of diagnosis to death or last follow up and were estimated using the Kaplan-Meier curves. The logrank test was used to compare overall survival (OS) between subgroups. Prognostic factors that would affect OS were analyzed by using univariate and multivariate Cox proportional hazard regression models adjusting for confounding variables. The statistical significance level was considered as $\mathrm{p}<0.05$. All statistical analyses were performed by using Statistical Package for Social Sciences (IBM, Chicago, USA).

\section{RESULTS}

\section{Patient Characteristics and Clinical Datas}

Data from 90 patients were analyzed. Median age at diagnosis was 59 years. Forty-two patients (53\%) were older than 60 years of age. Forty-four (49\%) of all patients enrolled to this study were male $46(51 \%)$. Female and sex ratio $(\mathrm{M} / \mathrm{F})$ was 0.96 . Median follow-up time was 31 months (range, 1 to 104 months). Forty-eight tumor samples were taken from lymph nodes and forty-two from extranodal sites. Twelve of the tumors were taken from the gastrointestinal system, 11 from Waldeyer's ring, 11 from the bones, two from the lungs and the remaining tumor samples each was from other organ sites. Forty-four of 58 cases (76\%) had high Ann-Arbor stage (III-IV). Twenty seven of 52 cases had poor R-IPI score and 31 of 52 cases had high or high/ intermediate NCCN-IPI scores.

No significant OS difference was observed when the cases grouped according to gender. Patients who were older than 60 years had significantly worse OS rates $(\mathrm{p}=0.001)$. No significant OS difference was detected for tumors taken from lymph nodes or extranodal sites $(\mathrm{p}=0.222)$, although, slightly better clinical course was observed in extranodal tumors. OS was better for low Ann-Arbor stages, R-IPI and NCCN-IPI scores that was statistically significant ( $\mathrm{p}=0.026, \mathrm{p}<0.001, \mathrm{p}<0.001$, respectively). 


\section{Immunohistochemical Analysis Results}

Of the 90 cases enrolled in the study, 19 cases showed MYC positivity and 53 cases showed BCL2 positivity. Among them 11 cases $(12.2 \%)$ were double-expressor lymphoma that stained both antibodies. It was also observed that there was no overexpression with either MYC or BCL2 antibodies in 20 patients that were defined as double-negative cases (Figures 1 and 2). The demographic,

a)

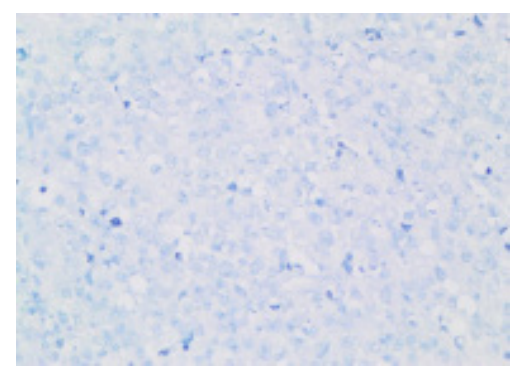

Figure 1. MYC $<40 \%$ (a), and $\geq 40 \%$ (b) immunoexpression(200X).

a)

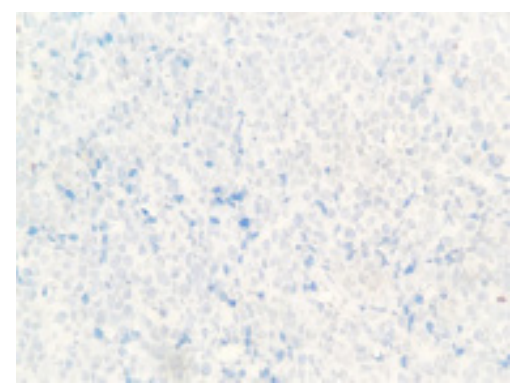

clinicopathologic and immunhistochemical data of DEL subgroup are summarized in Table I. Patients with MYC positivity displayed poorer OS than MYC negative cases $(p=0.018)$, whereas no significant difference was determined for BCL2 antibody $(\mathrm{p}=0.073)$. Double-expressor lymphoma cases staistically did not show any OS difference when compared with other cases $(p=0.169)$, albeit, these cases tended to show poor OS (Figure 3).

b)

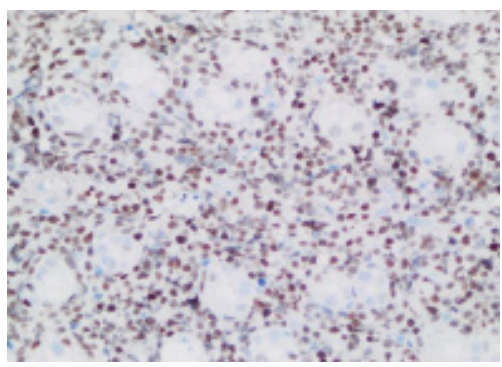

Figure 2. BCL2, $<50 \%$ (a), and $\geq 50 \%$ (b) immunoexpression (200X).

a)

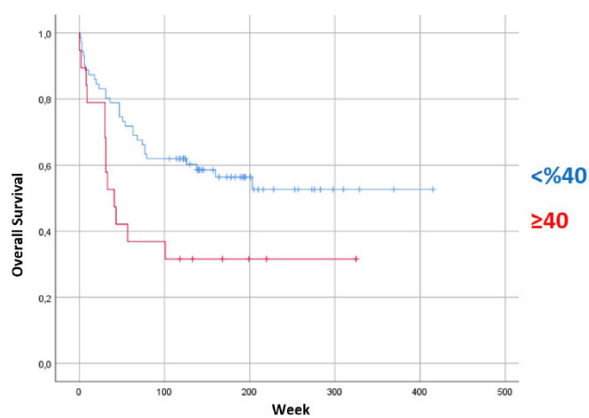

b)

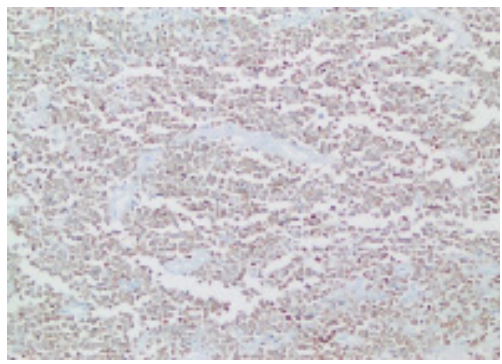

c)

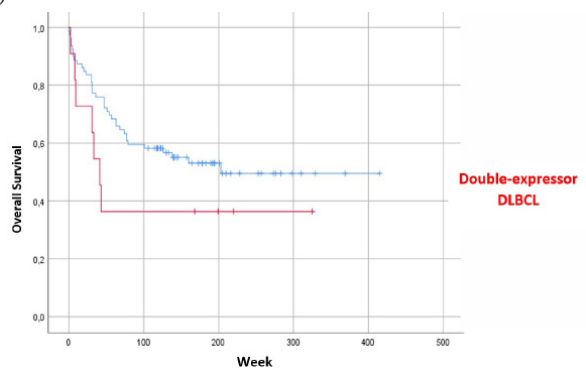

Figure 3. MYC expression-Overall survival (a), BCl2 expression-Overall survival (b), Double expressor lymphoma-Overall survival (c). 
When cases grouped as only MYC positive, only BCL2 positive, double-expressor lymphoma and double negative lymphoma; double negative cases showed markedly better OS than other three groups $(\mathrm{p}=0.015)$. There was no significant association between double-expressor lymphoma and the factors including sex, age and tumor localization, Ann-Arbor stage and R-IPI/ NCCN-IPI scores were distrubuted independently (Table I).

Table I. The demographic, clinicopathologic and immunohistochemical data of DEL subgroup

\begin{tabular}{|c|c|c|c|c|c|}
\hline \multicolumn{2}{|c|}{ Characteristics } & \multirow{3}{*}{$\begin{array}{c}\text { DEL }(\mathrm{n}=11) \\
4 \\
7\end{array}$} & \multirow{3}{*}{$\begin{array}{c}\begin{array}{c}\text { Non-DEL } \\
(\mathbf{n}=79)\end{array} \\
40 \\
39\end{array}$} & \multirow{3}{*}{$\begin{array}{l}\text { All } \\
44 \\
46\end{array}$} & \multirow{3}{*}{$\begin{array}{c}\text { p value } \\
0.375\end{array}$} \\
\hline Gender & Male & & & & \\
\hline & Female & & & & \\
\hline \multirow[t]{2}{*}{ Age } & $\leq 60$ & 4 & 38 & 42 & 0.229 \\
\hline & $>60$ & 7 & 41 & 48 & \\
\hline \multirow{2}{*}{$\begin{array}{l}\text { Tumor } \\
\text { location }\end{array}$} & Nodal & 4 & 44 & 48 & 0.229 \\
\hline & Extranodal & 7 & 35 & 42 & \\
\hline \multirow[t]{2}{*}{ AID } & $>\% 20$ & 1 & 15 & 16 & 0.421 \\
\hline & $\leq \% 20$ & 10 & 64 & 74 & \\
\hline \multirow[t]{2}{*}{ UNG } & $>\% 50$ & 3 & 14 & 17 & 0.449 \\
\hline & $\leq \% 50$ & 8 & 65 & 73 & \\
\hline \multirow[t]{2}{*}{ p53 } & $>\% 50$ & 8 & 40 & 48 & 0.169 \\
\hline & $\leq \% 50$ & 3 & 39 & 42 & \\
\hline \multirow{2}{*}{\begin{tabular}{|l|} 
Cell \\
origin
\end{tabular}} & GCB & 7 & 34 & 41 & 0.199 \\
\hline & Non-GBC & 4 & 45 & 49 & \\
\hline
\end{tabular}

DEL: Double-expressor lymphoma, AID: Activation-induced cytidine deaminase, UNG: uracil-DNA glycosylase, GBC: Germinal center B-cell

Cell of origin subtype was determined for all patients according to Hans algorithm. Forty-one cases (46\%) were GCB and 49 cases (54\%) were non-GCB subtypes. There was no significant prognostic difference among patients with GCB or non-GCB subtypes $(\mathrm{p}=0.707)$.

Cases were classified into two groups according to the cut-off value for AID antibody that was accepted as $20 \%$ staning of tumor cells. Sixteen cases showed immunreactivity for AID antibody in more than $20 \%$ of tumor cells, whereas 74 cases did not show any immunoreactivity for AID antibody. Cases were divided into two groups as: immunexpression for UNG antibody more or less than $50 \%$ of cells. Immunostaining in more than half of the neoplastic cells was observed in 17 cases (18.9\%). No significant difference was observed between groups for both antibodies in terms of overall survival $(p=0.330$ and $\mathrm{p}=0.559)$. In 42 cases $(46.7 \%)$, more than $50 \%$ of the neoplastic cells had p53 immunexpression and in 48 cases (53.3\%), less than $50 \%$ neoplastic cells had p53 immunexpression. There was no difference between these groups in terms of overall survival $(\mathrm{p}=0.740)$. The loss of expression with MLH1, MSH2, MSH6 and PMS2 was not observed in any tumor sample.
Multivariate analysis demonstrated that age, MYC expression and Ki67 expression were independent prognostic factors.

\section{DISCUSSION}

It was first reported that double-expressions of MYC and BCL2 in DLBCL were prognostic factors by earlier studies of Johnson et al. and Green et al. [5-6]. Numerous studies have contributed to our knowledge about these cases. DLBCL cases that show overexpression with both MYC and BCL2 antibodies with the cut-off values of $40 \%$ and $50 \%$ respectively, are defined as double-expressor lymphoma, according the last WHO lymphoma classification update $[3,15]$. It is advised to identify these cases because of the fact that DEL subset in DLBCL may be related with poor prognosis. Our study focused on immunohistochemical overexpression of MYC and BCL2 in DLBCL. Even though, there were differrent threshold values at earlier papers, $40 \%$ and 50\% cut-off values are accepted widely nowadays. Thereby, we determined DEL cases as parallel to the WHO suggestion. Incidence of the DEL subset in DLBCL was reported as between $20 \%-35 \%$ in previous studies with different cut-off values but the incidence of DEL cases in our study was $12.2 \%[15]$.

It has been stated that DEL subset is associated with a poor prognosis in DLBCL in many studies. Firstly, Johnson et al. and Green et al., reported that double-expressions of MYC and BCL2 were prognostic factors independent of genetic rearrangement of MYC and BCL2 genes [5,6]. However, our study showed that the association regarding the role of DEL may not be reliable. Our results suggested that DEL cases showed poor clinical courses, but these were not independent predictors of poor survival. Although, inadequate sample size and limited number of DEL cases may have limited our ability to detect significant prognostic differences, there are some other publications that support our data. Therefore, the role of DEL as prognostic subgroup is still controversial. In this study, we found that MYC expression in more than $40 \%$ of tumor cells detected by immunohistochemistry were associated with shorter OS in DLBCL irrespectively of the NCCN-IPI scores and Ann-Arbor stage. In multivariate analysis, we confirmed that MYC overexpression was an independent prognostic factor in DLBCL. On the other hand, BCL2 expression more than $50 \%$ of tumor cells showed no significant association with poor OS. Although, many studies support poor prognostic role of DEL subset, it will be better to confirm the role of DEL with further studies with large data-sets or meta-analyses. The other limitation of our analysis was the lack of FISH analysis, thus double-hit lymphoma subgroup which was associated with poor prognosis could not be determined. This made study population heterogeneous in terms of cell origin.

The affect of primary localization of tumor to OS is controversial, because of several reasons including classification problems and difficulties of determination of tumor location [17]. It is still a controversial issue whether Waldeyer's ring tumors are nodal or not. Some tumors may be located at or invade both nodal and extranodal sites and it is not easy to detect actual origin of the 
tumor. In this study, we detected the localization according to biopsy site and extranodal tumors, especially Waldeyer's ring and the bones tended to show better clinical course but that was not statistically significant. In the literature, studies do not have consensus about prognostic importance of primary tumor location. However, better outcomes in the head and neck DLBLCs are seen in some studies [18-21]. Patient age, AnnArbor stage and NCCN-IPI scores were important predictors of OS in our study as parallel to studies in literature [22-28]. Meanwhile, in our study, not reaching all IPI scores and stages of patients were also considered as a limitation.

The cases in our study were examined in two groups as GCB and non-GCB according to gene expression profiles. They were determined by immunohistochemical Hans algorithm methods [29]. There was no significant difference in overall survival between GCB and non-GCB groups in our study. Although, the Hans algorithm has an acceptable application validity, it is reported that its efficacy is limited in terms of identifying 10$15 \%$ of the cases, which cannot be classified by gene expression profiles, repeatability problems, application errors, and weak prognostic value [30-35].

AID and UNG are DNA base excision repair proteins that have important role during B-cell maturation. They create DNA breaks and mutations in physiological processes as somatic hypermutations and class switch recombination [36]. Since, these proteins take role in the formation and repair of DNA breaks, which cause translocations and may have a role in lymphoma etiopathogenesis. Immunostaining of the AID protein has been reported in several lymphomas, especially in DLBCL and Hodgkin's lymphoma. There are studies showing that AID immunostaining may be associated with poor prognosis in patients with chronic lymphocytic leukemia / small lymphocytic lymphoma (CLL / SLL) and follicular lymphoma. We have limited knowledge about the expression of UNG in DLBCL [9, 37-42]. The loss of UNG and mismatch repair proteins in murine patients have been shown to increase the rate of mutation and cause DLBCL-like disease, while only loss of UNG is shown to be protective [42]. In our study, there was no relation between the expression of AID/UNG proteins and overall survival or DEL status. Few publications indicate that the loss in the immunexpressions of mismatch repair proteins in DLBCL cases is not expected $[43,44]$. Consistently, we did not see any loss of these proteins in our study.

It is reported that overexpression of p53 protein may be associated with mutations in the p 53 gene and lymphomagenesis [45]. There are reports that show relationship between p53 expression and MYC protein expression and / or MYC gene translocation in DLBCL [10,46-48]. In some studies, increased p53 immunoexpression has been reported to be a poor prognostic factor in DLBCL $[46,47,49,50]$. In our study, there was no correlation between p53 expression and overall survival or DEL subgroup. The correlation between immunexpressions of p53 and MYC were solely observed.

In conclusion, our result did not demonstrate that doubleexpressor lymphoma was associated with poor outcomes, although, we showed a relationship between low overall survival and MYC expression. We did not detect any significant relation between AID, UNG and p53 immunexpressions with doubleexpressor lymphoma and overall survival. It had been concluded that mismatch repair proteins did not play a significant role in the pathogenesis of DLBCL due to the lack of loss of immunoexpression.

Acknowledgements: The authors would like to thank the physicians and other medical staff at Marmara University Hospital for their help, and many useful comments regarding this work.

\section{Compliance with Ethical Standards}

Ethical Approval: This study was approved by Marmara University Clinical Research Ethics Committee (Protocol number: 09.2016.578). All methods were performed in accordance with the relevant guidelines regulations.

Funding: This work was supported by Research Fund of the Marmara University. Project Number: SAG-CTUP-131.216.0526.

Conflict of Interest: The authors declare that they have no conflict of interest.

Authors' Contributions: Design: MHT, Supervision: TT, SB, Resources: MHT, SB, TE, TT, Materials: SB, TE, TT, Data collection and/or processing: MHT, SB, TE, TT, Analysis and/ or interpretation: MHT, SB, Writing manuscript: MHT, Critical review: SB.

\section{REFERENCES}

[1] Schmidt MT, Huang Q, Alkan S. Aggressive B-cell lymphomas: a review and practical approach for the practicing pathologist. Adv Anatomic Pathol 2015;22:168-80. doi: 10.1097/ PAP.000.000.0000000065

[2] Anderson JR, Armitage JO, Weisenburger DD. Epidemiology of the non-Hodgkin's lymphomas: distributions of the major subtypes differ by geographic locations. Non-Hodgkin's Lymphoma Classification Project. Ann Oncol 1998;9:717-20. doi: 10.1023/a:100.826.5532487

[3] Swerdlow SH. WHO classification of tumours of haematopoietic and lymphoid tissues. Lyon: International Agency for Research on Cancer; 2017.

[4] $\mathrm{Hu} \mathrm{S}$, Xu-Monette ZY, Tzankov A, et al. MYC/BCL2 protein coexpression contributes to the inferior survival of activated B-cell subtype of diffuse large B-cell lymphoma and demonstrates high-risk gene expression signatures: a report from The International DLBCL Rituximab-CHOP Consortium Program. Blood 2013;121:4021-31; quiz 250. doi: 10.1182/blood-2012-10-460063

[5] Johnson NA, Slack GW, Savage KJ, et al. Concurrent expression of MYC and BCL2 in diffuse large B-cell lymphoma treated with rituximab plus cyclophosphamide, doxorubicin, vincristine, and prednisone. J Clin Oncol 2012;30:3452-9. doi: 10.1200/JCO.2011.41.0985

[6] Green TM, Young KH, Visco C, et al. Immunohistochemical double-hit score is a strong predictor of outcome in patients 
with diffuse large B-cell lymphoma treated with rituximab plus cyclophosphamide, doxorubicin, vincristine, and prednisone. J Clin Oncol 2012;30:3460-7. doi: 10.1200/JCO.2011.41.4342

[7] Perry AM, Alvarado-Bernal Y, Laurini JA, et al. MYC and BCL2 protein expression predicts survival in patients with diffuse large B-cell lymphoma treated with rituximab. Br J Haematol 2014;165:382-91. doi: 10.1111/bjh.12763

[8] Salles G, de Jong D, Xie W, et al. Prognostic significance of immunohistochemical biomarkers in diffuse large B-cell lymphoma: a study from the Lunenburg Lymphoma Biomarker Consortium. Blood 2011;117:7070-8. doi: 10.1182/ blood-2011-04-345256

[9] Greiner A, Tobollik S, Buettner M, et al. Differential expression of activation-induced cytidine deaminase (AID) in nodular lymphocyte-predominant and classical Hodgkin lymphoma. J Pathol 2005;205:541-7. doi: 10.1002/path.1746

[10] Ramiro AR, Jankovic M, Callen E, et al. Role of genomic instability and p53 in AID-induced c-myc-Igh translocations. Nature 2006;440(7080):105-9. doi: 10.1038/nature04495

[11] Dorsett Y, Robbiani DF, Jankovic M, Reina-San-Martin B, Eisenreich TR, Nussenzweig MC. A role for AID in chromosome translocations between $\mathrm{c}$-myc and the $\operatorname{IgH}$ variable region. J Exp Med 2007;204:2225-32. doi: 10.1084/ jem.20070884

[12] Pasqualucci L, Bhagat G, Jankovic M, et al. AID is required for germinal center-derived lymphomagenesis. Nat Genet 2008;40:108-12. doi: 10.1038/ng.2007.35

[13] Liu M, Duke JL, Richter DJ, et al. Two levels of protection for the B cell genome during somatic hypermutation. Nature 2008;451(7180):841-5. doi: 10.1038/nature06547

[14] Liu M, Schatz DG. Balancing AID and DNA repair during somatic hypermutation. Trends Immunol 2009;30:173-81. doi: 10.1016/j.it.2009.01.007

[15] Swerdlow SH, Campo E, Pileri SA, et al. The 2016 revision of the World Health Organization classification of lymphoid neoplasms. Blood 2016;127:2375-90. doi: 10.1182/ blood-2016-01-643569

[16] Hans CP, Weisenburger DD, Greiner TC, et al. Confirmation of the molecular classification of diffuse large B-cell lymphoma by immunohistochemistry using a tissue microarray. Blood 2004;103:275-82. doi: 10.1182/blood-2003-05-1545

[17] López-Guillermo A, Colomo L, Jiménez M, et al. Diffuse large B-cell lymphoma: clinical and biological characterization and outcome according to the nodal or extranodal primary origin. J Clin Oncol 2005;23:2797-804. doi: 10.1200/JCO.2005.07.155

[18] Castillo JJ, Winer ES, Olszewski AJ. Sites of extranodal involvement are prognostic in patients with diffuse large B-cell lymphoma in the rituximab era: an analysis of the Surveillance, Epidemiology and End Results database. Am J Hematol 2014;89:310-4. doi: 10.1002/ajh.23638

[19] Moller MB, Pedersen NT, Christensen BE. Diffuse large B-cell lymphoma: clinical implications of extranodal versus nodal presentation-a population-based study of 1575 cases. Br J Haematol 2004;124:151-9. doi: 10.1046/j.13652141.2003.04749.x
[20] Lopez-Guillermo A, Colomo L, Jimenez M, et al. Diffuse large B-cell lymphoma: clinical and biological characterization and outcome according to the nodal or extranodal primary origin. J Clin Oncol 2005;23:2797-804. doi: 10.1200/JCO.2005.07.155

[21] Hui D, Proctor B, Donaldson J, et al. Prognostic implications of extranodal involvement in patients with diffuse large B-cell lymphoma treated with rituximab and cyclophosphamide, doxorubicin, vincristine, and prednisone. Leuk Lymphoma 2010;51:1658-67. doi: 10.3109/10428.194.2010.504872

[22] Armitage JO. A clinical evaluation of the International Lymphoma Study Group classification of non-Hodgkin's lymphoma. Blood 1997;89:3909-18. doi: 10.1182/blood. V89.11.3909

[23] Diebold J, Anderson JR, Armitage JO, et al. Diffuse large B-cell lymphoma: a clinicopathologic analysis of 444 cases classified according to the updated Kiel classification. Leuk Lymphoma 2002;43:97-104. doi: 10.1080/104.281.90210173

[24] Carella AM, de Souza CA, Luminari S, et al. Prognostic role of gender in diffuse large B-cell lymphoma treated with rituximab containing regimens: a Fondazione Italiana Linfomi/Grupo de Estudos em Molestias Onco-Hematologicas retrospective study. Leuk Lymphoma 2013;54:53-7. doi: 10.3109/10428.194.2012.691482

[25] Pregno P, Chiappella A, Bellò $M$, et al. Interim 18-FDG$\mathrm{PET} / \mathrm{CT}$ failed to predict the outcome in diffuse large B-cell lymphoma patients treated at the diagnosis with rituximab-CHOP. Blood 2012;119:2066-73. doi: 10.1182/ blood-2011-06-359943

[26] Le Guyader-Peyrou S, Orazio S, Dejardin O, Maynadie M, Troussard X, Monnereau A. Factors related to the relative survival of patients with diffuse large B-cell lymphoma in a population-based study in France: does socio-economic status have a role? Haematologica 2017;102:584-92. doi: 10.3324/ haematol.2016.152918

[27] Zhou Z, Sehn LH, Rademaker AW, et al. An enhanced International Prognostic Index (NCCN-IPI) for patients with diffuse large B-cell lymphoma treated in the rituximab era. Blood 2014;123:837-42. doi: 10.1182/blood-2013-09-524108

[28] Sehn LH, Berry B, Chhanabhai M, et al. The revised International Prognostic Index (R-IPI) is a better predictor of outcome than the standard IPI for patients with diffuse large B-cell lymphoma treated with R-CHOP. Blood 2007;109:185761. doi: 10.1182/blood-2006-08-038257

[29] Alizadeh AA, Eisen MB, Davis RE, et al. Distinct types of diffuse large B-cell lymphoma identified by gene expression profiling. Nature 2000;403(6769):503-11. doi: 10.1038/35000501

[30] Read JA, Koff JL, Nastoupil LJ, Williams JN, Cohen JB, Flowers CR. Evaluating cell-of-origin subtype methods for predicting diffuse large B-cell lymphoma survival: a meta-analysis of gene expression profiling and immunohistochemistry algorithms. Clin Lymphoma Myeloma Leuk 2014;14:460-7 e2. doi: 10.1016/j.clml.2014.05.002

[31] Gutierrez-Garcia G, Cardesa-Salzmann T, Climent F, et al. Gene-expression profiling and not immunophenotypic algorithms predicts prognosis in patients with diffuse large 
B-cell lymphoma treated with immunochemotherapy. Blood 2011;117:4836-43. doi: 10.1182/blood-2010-12-322362

[32] Barrans S, Crouch S, Smith A, Turner K, Owen R, Patmore R, et al. Rearrangement of MYC is associated with poor prognosis in patients with diffuse large B-cell lymphoma treated in the era of rituximab. J Clin Oncol 2010;28:3360-5. doi: 10.1200/ JCO.2009.26.3947

[33] Ott G, Ziepert M, Klapper W, et al. Immunoblastic morphology but not the immunohistochemical GCB/nonGCB classifier predicts outcome in diffuse large B-cell lymphoma in the RICOVER-60 trial of the DSHNHL. Blood 2010;116:4916-25. doi: 10.1182/blood-2010-03-276766

[34] Hong J, Park S, Park J, et al. CD99 expression and newly diagnosed diffuse large B-cell lymphoma treated with rituximab-CHOP immunochemotherapy. Annals Hematol 2012;91:1897-906. doi: 10.1007/s00277.012.1533-Z

[35] Seki R, Ohshima K, Fujisaki T, et al. Prognostic impact of immunohistochemical biomarkers in diffuse large B囚cell lymphoma in the rituximab era. Cancer Sci 2009;100:1842-7. doi: 10.1111/j.1349-7006.2009.01268.x

[36] Stavnezer J. Complex regulation and function of activationinduced cytidine deaminase. Trends Immunol 2011;32:194201. doi: 10.1016/j.it.2011.03.003

[37] Willenbrock K, Renne C, Rottenkolber M, et al. The expression of activation induced cytidine deaminase in follicular lymphoma is independent of prognosis and stage. Histopathology 2009;54:509-12. doi: 10.1111/j.13652559.2009.03241.x

[38] Shi Y, Zhao X, Durkin L, Rogers HJ, Hsi ED. Aberrant activation-induced cytidine deaminase expression in Philadelphia chromosome-positive B-cell acute lymphoblastic leukemia. Hum Pathol 2016;52:173-8. doi: 10.1016/j. humpath.2016.01.008

[39] Lossos IS, Levy R, Alizadeh AA. AID is expressed in germinal center B-cell-like and activated B-cell-like diffuse large-cell lymphomas and is not correlated with intraclonal heterogeneity. Leukemia 2004;18:1775-9. doi: 10.1038/ sj.leu.2403488

[40] Greeve J, Philipsen A, Krause K, et al. Expression of activation-induced cytidine deaminase in human B-cell nonHodgkin lymphomas. Blood 2003;101:3574-80. doi: 10.1182/ blood-2002-08-2424
[41] Engels K, Jungnickel B, Tobollik S, Hansmann M L, Kriener S, Willenbrock K. Expression of activation-induced cytidine deaminase in malignant lymphomas infiltrating the bone marrow. Appl Immunohisto M M 2008;16:521-9. doi: 10.1097/ PAI.0b013e3181758ce5

[42] Gu X, Booth CJ, Liu Z, Strout MP. AID-associated DNA repair pathways regulate malignant transformation in a murine model of BCL6-driven diffuse large B-cell lymphoma. Blood 2016;127:102-12. doi: 10.1182/blood-2015-02-628164

[43] Rossi D, Rasi S, Di Rocco A, et al. The host genetic background of DNA repair mechanisms is an independent predictor of survival in diffuse large B-cell lymphoma. Blood 2011;117:2405-13. doi: 10.1182/blood-2010-07-296244

[44] Couronne L, Ruminy P, Waultier-Rascalou A, et al. Mutation mismatch repair gene deletions in diffuse large B-cell lymphoma. Leuk Lymphoma 2013;54:1079-86. doi: 10.3109/10428.194.2012.739687

[45] Lu TX, Young KH, Xu W, Li JY. TP53 dysfunction in diffuse large B-cell lymphoma. Crit Rev Oncol Hematol 2016;97:4755. doi: 10.1016/j.critrevonc.2015.08.006

[46] Wang XJ, Medeiros LJ, Bueso-Ramos CE, et al. P53 expression correlates with poorer survival and augments the negative prognostic effect of MYC rearrangement, expression or concurrent MYC/BCL2 expression in diffuse large B-cell lymphoma. Mod Pathol 2017;30:194-203. doi: 10.1038/ modpathol.2016.178

[47] Li S, Weiss VL, Wang XJ, et al. High-grade B-cell lymphoma with MYC rearrangement and without BCL2 and BCL6 rearrangements is associated with high P53 expression and a poor prognosis. Am J Surg Pathol 2016;40:253-61. doi: 10.1097/PAS.000.000.0000000542

[48] Tessoulin B, Eveillard M, Lok A, et al. p53 dysregulation in B-cell malignancies: More than a single gene in the pathway to hell. Blood Rev 2017;31:251-9. doi: 10.1016/j.blre.2017.03.001

[49] Fiskvik I, Beiske K, Delabie J, et al. Combining MYC, BCL2 and TP53 gene and protein expression alterations improves risk stratification in diffuselarge B-celllymphoma. Leuk Lymphoma 2015;56:1742-9. doi: 10.3109/10428.194.2014.970550

[50] Schiefer AI, Kornauth C, Simonitsch-Klupp I, et al. Impact of single or combined genomic alterations of TP53, MYC, and BCL2 on survival of patients with diffuse large B-cell lymphomas: A retrospectivecohort study. Medicine(Baltimore) 2015;94:e2388. doi: 10.1097/MD.000.000.0000002388 